http://jmscr.igmpublication.org/home/

ISSN (e)-2347-176x ISSN (p) 2455-0450

crossref DOI: https://dx.doi.org/10.18535/jmscr/v7i10.136

Journal Of Medical Science And Clinical Research

IGM Publication

An official Publication of IGM Publication

\title{
Chorea Associated with Non Ketotic Hyperglycemia (Diabetic Striatopathy) in Elderly Female - A Case Report
}

Authors

Dr Balakumar.J', Prof. Dr S.Balasubramaniyan.M.D², Dr N.Paari M.D. ${ }^{3}$

${ }^{1}$ Post Graduate, Department of General Medicine, Rajah Muthiah Medical College \& Hospital, Annamalai University, Chidambaram, India - 608002

${ }^{2}$ Professor, Department of General Medicine, Rajah Muthiah Medical College \& Hospital, Annamalai University, Chidambaram, India - 608002

${ }^{3}$ Assistant Professor, Department of General Medicine, Rajah Muthiah Medical College \& Hospital, Annamalai University, Chidambaram, India - 608002

\section{Introduction}

The term "diabetic striatopathy" is characterized by the presence of a high signal on MRI confined to the striatum with contralateral movement disorder. It is commonly associated with type 2 Diabetes mellitus and rarely seen in type 1 diabetes mellitus. Many cases at the time of admission had many clinical features similar to non-ketotic hyperglycemia ${ }^{1}$. Rarely, patients have bilateral lesions with bilateral chorea. Even though the precise mechanism is unidentified, the primary chronic focal cerebrovascular disease in Diabetes Mellitus may be the cause for acute dysfunction of blood brain barrier ${ }^{2}$.

\section{Case Report}

58 year old female was brought to emergency department with sudden onset of involuntary movements involving both left upper limb and lower limb, characterized by jerky movements since 4 hours. No history of altered sensorium or any motor weakness, No history of syncope, No features suggestive of cranial nerve involvement. Patient is a known case of coronary artery disease and type 2 diabetes mellitus for 7 years on oral hypoglycemic agent.

On examination patient was conscious, oriented Pulse rate of 80/min, Blood Pressure - 140/90 mm Hg, RR- 18/min. patient had clumpsy gait, she had uncontrollable involuntary chorea like movement in left upper and lower limb and her muscle tone and power were normal, systemic examination was normal,

\section{Investigation}

\begin{tabular}{|l|c|}
\hline Random blood sugar & $414 \mathrm{mg} / \mathrm{dl}$ \\
\hline Blood urea & $37 \mathrm{mg} / \mathrm{dl}$ \\
\hline Seum creatinine & $1.4 \mathrm{mg} / \mathrm{dl}$. \\
\hline Serum sodium serum & $142 \mathrm{mmo} / \mathrm{l}$ \\
\hline $\begin{array}{l}\text { Calculated } \\
\text { osmalilty }\end{array}$ & 309 \\
\hline Plasma acetone & negative \\
\hline
\end{tabular}

MRI BRAIN (fig-1) shows T1, T2 hyperintensities noted in right basal ganglia and right periventicular corona radiata with diffusion restriction, suggestive of non ketotic hyperglycemic hemichorea. With this clinical presentation, nonketotic hyperglycemia, and MRI finding, a diagnosis of diabetic striatopathy was 
made. Blood sugar was managed with insulin. After adequate control of blood sugar on second day the involuntary movement has resolved.

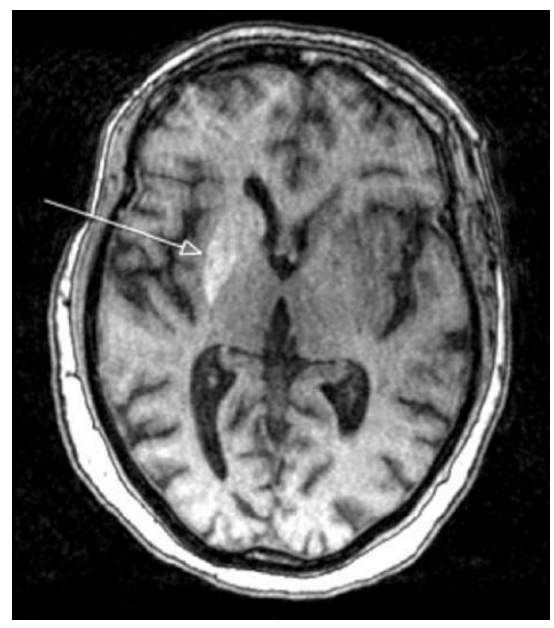

Fig-1: MRI Brain

\section{Discussion}

Diabetic striatopathy has been implicated as rare and fatal manifestation of diabetes mellitus. It is more common in elderly women of asia and they usually manifest as hemiballism-hemichorea.

Hemiballismus is an unilateral ballimus, an involuntary hyperkinetic movement disorder comprising of large amplitude vigorous irregular movements of the body. Disorders like stroke, diabetic striatopathy, malignancy, infection, Wilson's disease, and grave's disease results in this type of neurological manifestation ${ }^{3}$.

Eventhough the incidence of striatopathy is in frequent, precise earlier identification of a hyperglycemia-induced hemiballism-hemichorea is vital as the symptoms are reversed with glycemic correction. Diagnosis mainly depends on arterial blood gas analysis and radiological findings. Blood glucose and $\mathrm{HbA1C}$ levels are usually increasesd and it indicates poor diabetic control. Hyperattenuation on CT scan and hyperintensity on T1-weighted MRI in the basal ganglia are the significant radiological features of diabetic striatopathy.

Widely established pathophysiological mechanism in diabetic striatopathy involves hyperviscosity leading to local tissue hypoperfusion, depletion of Gamma- Aminobutyric acid and accumulation of manganese- containing gemistocytes in the basal ganglia which typically appear as T1 hyperintense lesions.

Striatal hyper intensity on CT and MRI in the initial stage of non-ketotic hyperglycemia helps in the early identification and treatment. The choreaballismus is completely reversible. Radiological findings may return to normal or persist after clinical recovery ${ }^{4}$.

\section{References}

1. Abe Y, Yamamoto T, Soeda T, Kumagai T, Tanno Y, Kubo J, et al. Diabetic striatal disease: clinical presentation, neuroimaging, and pathology. Intern Med. 2009; 48: 1135-41.

2. Lai PH, Tien RD, Chang MH, Teng MM, Yang CF, Pan HB, et al. Chorea-ballismus with nonketotic hyperglycemia in primary diabetes mellitus. AJNR Am J Neuroradiol. 1996; 17:1057-64.

3. Bathla G, Policeni B, Agarwal A (2014) Neuroimaging in patients with abnormal blood glucose levels. AJNR Am J Neuroradiol 35(5):830-840.

4. Battisti C, Forte F, Rubenni E, Dotti MT, Bartali A, Gennari P, et al. Two cases of hemichorea-hemiballism with nonketotic hyperglycemia: A new point of view. Neurol Sci. 2009;30:179-83. 